\title{
Rastreo de familiares de una paciente con miocardiopatía hipertrófica obstructiva
}

\section{Eliza Kaori Uenishi*, Tarek Fakih Hachen, Clara I. Arias Barrera y Carlos A. Ariza-La Rotta}

\author{
Fundación Cardiovascular de Colombia-Instituto del Corazón, Santa Marta, Colombia
}

Recibido el 27 de septiembre de 2016; aceptado el 15 de febrero de 2017

Disponible en Internet el 1 de agosto de 2017

\section{PALABRAS CLAVE \\ Miocardiopatía \\ hipertrófica familiar; \\ Muerte súbita}

\section{KEYWORDS}

Familial hypertrophic myocardiopathy;

Sudden death

\begin{abstract}
Resumen
Introducción: La miocardiopatía hipertrófica familiar es la modalidad hereditaria autosómica dominante de la miocardiopatía hipertrófica, de penetrancia incompleta y expresión variable.

Se exponen los estudios realizados a los familiares de un caso índice de miocardiopatía hipertrófica obstructiva, dado un $50 \%$ de probabilidad de padecer la enfermedad.

Material y métodos: A partir de un caso índice se reclutaron familiares (hijos y hermanos) del caso índice, quienes fueron invitados a realizarse electrocardiograma, ecocardiograma transtorácico, prueba de esfuerzo y Holter cardíaco de manera voluntaria. Se aplicó una encuesta semiestructurada para obtener datos sociodemográficos, antecedentes personales, familiares y hábito de práctica de actividad física. Para el diagnóstico se siguieron los criterios utilizados por la guía de la Sociedad española de cardiología.

Resultados: En los 11 casos evaluados se identificaron 4 miembros de la familia con criterios diagnósticos de miocardiopatía hipertrófica y 5 con hipertensión arterial.

Conclusión: Con el estudio de los familiares de la paciente índice se logró descubrir la afectación de esta enfermedad en 2 familiares de primer grado y en 2 familiares de segundo grado, con predominancia para el sexo masculino (3:1); en la paciente femenina se encontró, además, hipertensión arterial y miocardiopatía hipertrófica asociadas a fibrilación auricular. Ninguno de ellos tuvo historia personal de síncope o resucitación de muerte súbita.

( $) 2017$ Sociedad Colombiana de Cardiología y Cirugía Cardiovascular. Publicado por Elsevier España, S.L.U. Este es un artículo Open Access bajo la licencia CC BY-NC-ND (http:// creativecommons.org/licenses/by-nc-nd/4.0/).
\end{abstract}

Family tracing of a patient with hypertrophic obstructive miocardiomyopathy

Abstract

Introduction: Familial hypertrophic myocardiopathy is a hereditary autosomal dominant trait of hypertrophic myocardiopathy, of incomplete penetrance and variable expression.

\footnotetext{
* Autor para correspondencia.

Correo electrónico: ezkaori@yahoo.com.br (E.K. Uenishi).
} 
Studies carried out on the family of an index case of hypertrophic obstructive myocardiopathy gave a $50 \%$ probability of suffering from the disease.

Material and methods: Family members (children, brothers and sisters) of an index case were recruited and were invited to volunteer to have a transthoracic electrocardiogram, stress test, and Holter test performed. A semi-structured questionnaire was completed in order to obtain sociodemographic data, personal and family history, and physical activity habits. The criteria used in the Spanish Cardiology Society guidelines were followed to make the diagnosis.

Results: In the 11 cases evaluated, 4 members of the family were identified with diagnostic criteria of hypertrophic myocardiopathy, and 5 with arterial hypertension.

Conclusions: With the study of the family members of the index patient, it was discovered that this disease affected 2 first degree and 2 second degree family members, with a predominance of male gender (3:1). The female case was also found to have arterial hypertension and hypertrophic cardiomyopathy associated with atrial fibrillation. None of them had a personal history of syncope or sudden death resuscitation.

(C) 2017 Sociedad Colombiana de Cardiología y Cirugía Cardiovascular. Published by Elsevier España, S.L.U. This is an open access article under the CC BY-NC-ND license (http:// creativecommons.org/licenses/by-nc-nd/4.0/).

\section{Introducción}

La miocardiopatía hipertrófica es una enfermedad miocárdica primaria, caracterizada fundamentalmente por hipertrofia ventricular izquierda, en ausencia de alguna enfermedad cardíaca o sistémica capaz de producirla, como estenosis valvular aórtica, hipertensión arterial sistémica o cardiopatía congénita. La miocardiopatía hipertrófica familiar es la forma hereditaria autosómica dominante de la miocardiopatía hipertrófica, de penetrancia incompleta y expresión variable. La heterogeneidad genética podría explicar las variaciones en el fenotipo, en las manifestaciones clínicas y en el pronóstico.

La prevalencia en adultos oscila entre 0,02-0,23\% en el mundo y específicamente en la población pediátrica, la incidencia anual es de $0,3-0,5 / 100.000^{1}$, con ligera predominancia en el sexo masculino. Desde el punto de vista morfológico, el hallazgo más importante es la hipertrofia miocárdica. Adicionalmente, hay una variedad de presentaciones anatómicas, entre ellas septal, apical, medio-ventricular y lateral, aunque también puede ser concéntrica.

La mitad de los pacientes son asintomáticos y se diagnostican de forma casual mediante un electrocardiograma o una exploración rutinaria. La muerte súbita puede ser la primera manifestación, sobre todo en jóvenes (de 14 a 35 años) y en la mayoría de los casos durante la práctica deportiva que requiere un ejercicio físico intenso ${ }^{1,2}$ o en pacientes con evolución estable; es extremadamente rara en niños menores de 5 años $^{3}$. En la literatura la incidencia de muerte súbita varía de 0,5 a $1 \%$ al año ${ }^{4}$. Los factores de riesgo más relacionados con la muerte súbita son: edad menor a 30 años, historia familiar de muerte súbita, anomalías genéticas asociadas a una alta prevalencia de muerte súbita, antecedente de parada cardíaca recuperada, síncope recurrente en jóvenes, bradiarritmias y taquicardia ventricular sostenida o taquicardia supraventricular.
Los síntomas clínicos más relevantes son disnea, dolor torácico, presíncope o síncope. La presencia de al menos un factor de riesgo, obliga el estudio del potencial arritmogénico $y$, de ser posible, también genético. El planteamiento inicial se centra en la prevención de la aparición de complicaciones y la estratificación del pronóstico de la enfermedad.

El ecocardiograma es considerado como la técnica de elección (recomendación clase I) tanto para el diagnóstico de la enfermedad, como para su seguimiento. Es el estudio complementario más utilizado en la actualidad para la evaluación clínica de los pacientes con miocardiopatía hipertrófica, pues permite estudiar aspectos anatómicos, alteraciones estructurales, funcionales y hemodinámicas, así como anomalías de distensibilidad y relajación ventricular típicos de estos pacientes, sin olvidar la evaluación de la respuesta al tratamiento y el control evolutivo de los casos diagnosticados. El ecocardiograma sirve además para evaluar los parámetros que contribuyen en la identificación del paciente con riesgo alto de muerte súbita (fig. 1).

Los criterios diagnósticos utilizados para determinar la miocardiopatía hipertrófica en adultos de la guía de la Sociedad Europea de Cardiología de $2014^{1}$ son grosor de la pared $\geq 15 \mathrm{~mm}$ en uno o más segmentos miocárdicos del ventrículo izquierdo (determinado por cualquier técnica de imagen), que no puede explicarse únicamente por condiciones de carga. El diagnóstico clínico en familiares de primer grado de pacientes con enfermedad indiscutible, se basa en el aumento del grosor del ventrículo izquierdo $\geq 13 \mathrm{~mm}$. El gradiente en el tracto de salida del ventrículo izquierdo se considera severo si es $\geq 50 \mathrm{~mm} \mathrm{Hg}$.

El electrocardiograma estándar de 12 derivaciones puede ser normal en el $6 \%$ de los casos, pero en general muestra una combinación variable de hipertrofia ventricular izquierda, anomalías de las ondas T y ST y ondas Q patológicas.

Los factores ecocardiográficos de peor pronóstico son: grosor de la pared ventricular (> $20 \mathrm{~mm})$, gradiente sistólico 


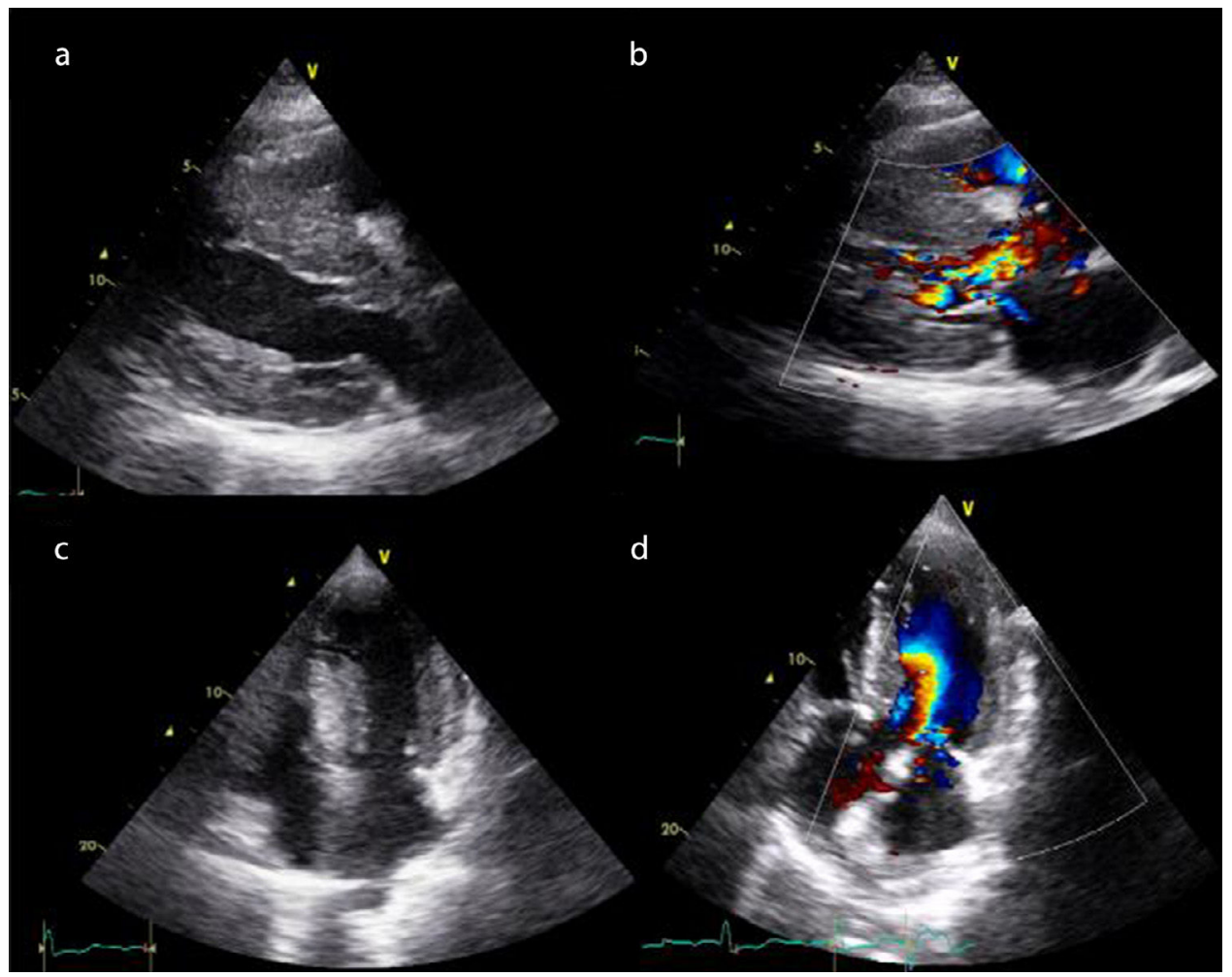

Figura 1 Imágenes de ecocardiografía bidimensional de paciente con miocardiomiopatía hipertrófica, proyección (a): paraesternal longitudinal,(b) paraesternal logintudinal con Doppler color que muestra insuficiencia mitral de grado leve y aceleración del flujo en el tracto de salida de ventrículo izquierdo, (c) apical cuatro cámaras, (d) apical cuatro cámaras con Doppler color.

máximo en el tracto de salida del ventrículo izquierdo (> $50 \mathrm{~mm} \mathrm{Hg}$ ), disfunción sistólica asociada a hipertrofia ventricular izquierda y análisis de la disfunción diastólica del ventrículo izquierdo.

Una vez hecho el diagnóstico, se evalúa el riesgo de muerte súbita. En la actualidad se adopta el modelo de predicción de riesgo de muerte súbita recomendado por la Sociedad Europea de Cardiología: HCM Risk-SCD ${ }^{1}$, score que tiene como propósito establecer el riesgo individual de muerte súbita en los próximos cinco años. Utiliza como parámetros la edad, el gradiente obstructivo en reposo y con maniobra de Valsalva, la evidencia de taquicardia ventricular no sostenida en el Holter, el máximo grosor ventricular, el diámetro anteroposterior de la aurícula izquierda y el síncope inexplicado. La guía propone que en un riesgo calculado superior a $6 \%$, se considera el implante de un desfibrilador (recomendación clase Ila), mientras que en un riesgo del 4 al 6\% se recomienda evaluar la implantación de forma individual (clase IIb) ${ }^{1}$. Pese a que varios estudios validan este modelo de predicción de riesgo clínico para muerte súbita a favor ${ }^{3,5,6}$, el estudio de Maron et al. evaluó la fidedignidad de este modelo estadístico y demostró la poca fiabilidad para la predicción de eventos futuros de muerte súbita en estos pacientes en 5 años $^{7}$. De modo que se requiere una evaluación pormenorizada y calcular una estimación del riesgo individual para contribuir al proceso de toma de decisiones clínicas, así como un estudio multicéntrico para la validación de este puntaje a fin de mejorar la estratificación del riesgo de los pacientes con miocardiopatía hipertrófica.
Según la guía de miocardiopatía hipertrófica de la Sociedad Europea de Cardiología de 2014 ${ }^{1}$, es esencial (recomendación clase I) hacer una evaluación clínica completa (incluidos electrocardiograma y ecocardiograma) en todos los familiares de primer grado de un caso índice. Como medidas preventivas generales se recomienda la supresión del ejercicio físico a nivel de competición (recomendación clase l) a los pacientes con peor pronóstico ecocardiográfico ${ }^{1}$.

Ya que la miocardiopatía hipertrófica es una enfermedad de transmisión autosómica, el diagnóstico es fundamental. La sospecha clínica, el estudio y el seguimiento de los familiares de primer grado de pacientes afectados, son las vías esenciales de detección precoz para evitar la pérdida adicional de vidas. Por esta razón, a continuación se exponen los estudios realizados a los familiares de un caso índice de miocardiopatía hipertrófica obstructiva, dada la probabilidad del $50 \%$ de padecer la enfermedad.

\section{Metodología}

A partir de un caso índice (paciente con diagnóstico comprobado de miocardiopatía hipertrófica) atendido en la Fundación Cardiovascular de Colombia - Instituto del Corazón de Santa Marta, se asignó un número para identificar cada miembro de la familia a ser estudiado. Se realizó reclutamiento de los hijos y hermanos, para un total de 11 individuos. Fueron invitados a practicarse de manera voluntaria exámenes diagnósticos como: electrocardiograma de 12 derivaciones, ecocardiograma Doppler 
modo $\mathrm{M}$ y bidimensional transtorácico, prueba de esfuerzo y Holter cardíaco de 24 horas, en formato de cortesía.

En la recolección de la información se aplicó una encuesta semiestructurada para obtener datos sociodemográficos, antecedentes personales y familiares e información sobre el hábito de practicar actividad física. La encuesta fue diligenciada personalmente a cada individuo (anexo 1).

Las imágenes ecocardiográficas se adquirieron en equipo Vivid S5 (General Electric ${ }^{\oplus}$ ), utilizando transductor de banda larga (S3), realizado por un único médico cardiólogo con entrenamiento específico en ecocardiografía. Se hizo estudio ecocardiográfico completo de acuerdo con las recomendaciones de la Sociedad Americana de Ecocardiografía ${ }^{8}$. Se midieron los tres segmentos (basal, medio y apical) en todas las paredes miocárdicas a través de modo bidimensional en eje paraesternal, longitudinal y apical en 4 y 2 cámaras y se usó Doppler continuo para la medición del gradiente del tracto de salida del ventrículo izquierdo en reposo y con maniobra de Valsalva.

El electrocardiograma de 12 derivaciones estándar fue tomado por una auxiliar de enfermería entrenada, con el electrocardiógrafo Autocardiner modelo FCP-2155 (Fukuda Denshi ${ }^{\oplus}$ ) y fue interpretado por un único cardiólogo.

Se usó el Holter CardioScan ${ }^{\otimes}$ dms-300, el cual fue instalado por un funcionario entrenado, que además dio orientaciones a los pacientes para reportar los síntomas en un formato específico; la lectura fue hecha por un único cardiólogo.

Por último, la prueba de esfuerzo fue practicada por un cardiólogo mediante el equipo Trackmaster ${ }^{\oplus}$ modelo TMX 428110, utilizando el protocolo de Bruce y fue interrumpida al manifestar síntomas o alcanzar la frecuencia cardíaca predeterminada.

Los criterios diagnósticos utilizados para determinar la miocardiopatía hipertrófica en adultos se basaron en la guía de la ESC 20141.

\section{Resultados}

El paciente índice (N. 1) es de sexo femenino, de 53 años de edad, casada y madre de tres hijos. Tiene diagnóstico de miocardiopatía hipertrófica hace 7 años, insuficiencia mitral severa y gradiente en el tracto de salida del ventrículo izquierdo de $84 \mathrm{~mm} \mathrm{Hg}$. Reportó ser hipertensa hace 5 años. Cuenta con historia de muerte súbita familiar (prima) y personal. Desde hace 3 años usa implante de cardiodesfibrilador bicameral AAIDDD (Medtronic virtuoso ${ }^{\circledR}$ ) y recibe como tratamiento médico amiodarona y betabloqueador. Refirió síntomas como falta de aire, dolor precordial, fatiga, palpitaciones y mareo. Es vendedora ambulante, camina varias horas al día y no practica ningún deporte. Presentó un episodio de muerte súbita hace 2 meses, del que fue resucitada por el cardiodesfibrilador. Durante la valoración se registró hipertensión arterial $(176 / 91 \mathrm{~mm} \mathrm{Hg})$, frecuencia cardíaca de 60 latidos por minuto, peso: $65,7 \mathrm{~kg}$ y talla: $1,56 \mathrm{~cm}$.

No se contó con muchos datos sobre los familiares de los padres de la paciente índice. Se confirmó que el papá falleció a los 80 años de edad por causa desconocida y que la mamá está viva y sana. Los tres tíos maternos fallecieron. No se tuvo conocimiento sobre la familia paterna.

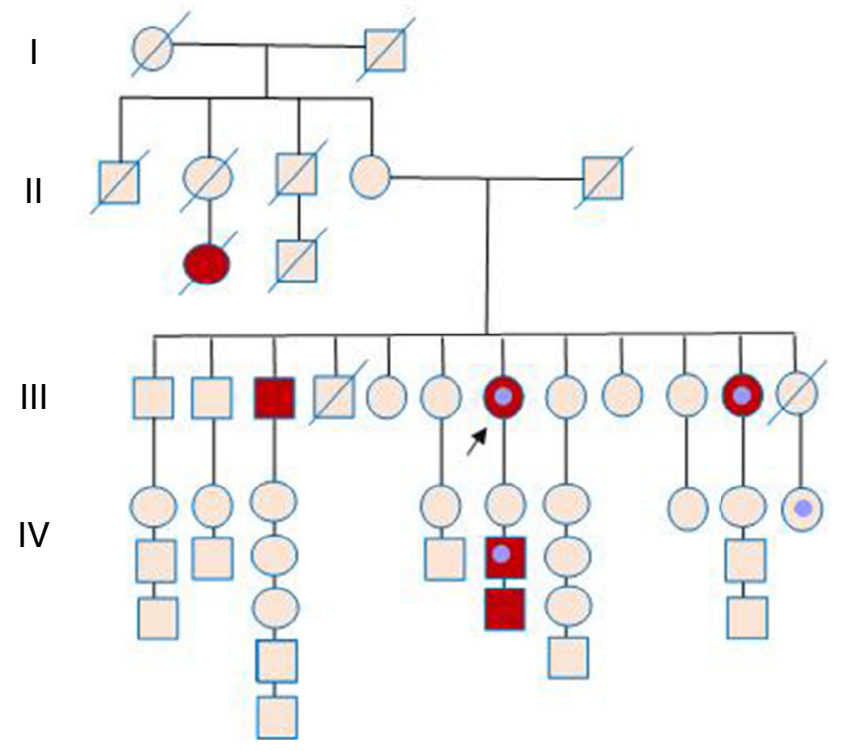

Figura 2 Árbol genealógico familiar. Círculo: mujer. Cuadrado: hombre. Símbolos rojos: miembros de la familia afectados de miocardiomiopatía/muerte súbita. Símbolos marrones: miembros fallecidos. Flecha: paciente índice. Símbolos que encierran un círculo: miembros portadores de dispositivo implantado (marcapaso/cardiodesfibrilador).

Tiene 11 hermanos, de los cuales 8 se incluyeron en el estudio. Entre estos parientes del segundo grado se excluyeron 3 debido a rechazo a realizarse los exámenes y 2 por haber fallecido, uno al año de edad (víctima de quemaduras) y otra a los 46 años de edad por causa desconocida, siendo sintomática por dolor precordial, mareo y palpitaciones y con una hija portadora de marcapaso, por causa no aclarada. Los 3 hijos de la paciente índice también fueron incluidos en el estudio y en 2 de ellos se diagnosticó la enfermedad (fig. 2).

Se tabularon los datos sociodemográficos, las medidas antropométricas, los parámetros clínicos y el hábito de actividad física de la población estudiada, Cinco de los familiares tienen el hábito de practicar actividad física, es decir, no son sedentarios; pero ninguno realiza ejercicio físico intenso (tabla 1).

A través del estudio de los familiares de la paciente índice, se identificaron 4 miembros de la familia con criterios diagnósticos de miocardiopatía hipertrófica. Se logró determinar 2 casos en familiares de primer grado (los pacientes números 2 y 3 ) siendo el número 3 portador conocido de miocardiopatía hipertrófica no obstructiva con cardiodesfibrilador implantable y en seguimiento por Electrofisiología, además 2 casos en familiares de segundo grado (los pacientes números 8 y 13). También se hallaron 5 pacientes con hipertensión arterial, de los cuales se diagnosticó un caso nuevo; excepto el paciente número 11, en todos se evidenció hipertrofia ventricular izquierda en el ecocardiograma. En 3 individuos se sospechó isquemia miocárdica por la prueba de esfuerzo. La paciente número 13, además de hipertensión arterial, presentaba criterios diagnósticos de miocardiopatía hipertrófica, con grosor máximo de la pared del ventrículo izquierdo de $25 \mathrm{~mm}$, y es portadora de 
Tabla 1 Datos clínicos y demográficos

\begin{tabular}{|c|c|c|c|c|c|c|c|c|c|c|c|}
\hline \multirow[t]{2}{*}{ Variables } & \multicolumn{11}{|c|}{ Identificación de los pacientes estudiados } \\
\hline & 2 & 3 & 4 & 5 & 6 & 7 & 8 & 10 & 11 & 12 & 13 \\
\hline $\begin{array}{l}\text { Sexo } \\
\text { Edad } \\
\text { Grado de parentesco }\end{array}$ & $\begin{array}{l}\text { Femenino } \\
20 \\
\mathrm{Hija}\end{array}$ & $\begin{array}{l}\text { Masculino } \\
25 \\
\text { Hijo }\end{array}$ & $\begin{array}{l}\text { Masculino } \\
23 \\
\text { Hijo }\end{array}$ & $\begin{array}{l}\text { Femenino } \\
60 \\
\text { Hermana }\end{array}$ & $\begin{array}{l}\text { Femenino } \\
47 \\
\text { Hermana }\end{array}$ & $\begin{array}{l}\text { Femenino } \\
71 \\
\text { Hermana }\end{array}$ & $\begin{array}{l}\text { Masculino } \\
66 \\
\text { Hermano }\end{array}$ & $\begin{array}{l}\text { Masculino } \\
64 \\
\text { Hermano }\end{array}$ & $\begin{array}{l}\text { Femenino } \\
51 \\
\text { Hermana }\end{array}$ & $\begin{array}{l}\text { Masculino } \\
62 \\
\text { Hermano }\end{array}$ & $\begin{array}{l}\text { Femenino } \\
58 \\
\text { Hermana }\end{array}$ \\
\hline $\begin{array}{l}\text { Estado civil } \\
\text { Número de hijos } \\
\text { Enfermedades asociadas }\end{array}$ & $\begin{array}{l}\text { Soltero } \\
0 \\
-\end{array}$ & $\begin{array}{l}\text { Soltero } \\
0 \\
\text { Miocardiopatía } \\
\text { hipertrófica }\end{array}$ & $\begin{array}{l}\text { Soltero } \\
0 \\
a_{-}\end{array}$ & $\begin{array}{l}\text { Casada } \\
4 \\
\text { Hipertensión } \\
\text { arterial }\end{array}$ & $\begin{array}{l}\text { Casada } \\
2 \\
-\end{array}$ & $\begin{array}{l}\text { Casada } \\
1 \\
\text { Hipertensión } \\
\text { arterial }\end{array}$ & $\begin{array}{l}\text { Casado } \\
5 \\
-\end{array}$ & $\begin{array}{l}\text { Soltero } \\
0 \\
\text { Dislipidemia }\end{array}$ & $\begin{array}{l}\text { Soltero } \\
0 \\
\text { Hipertensión } \\
\text { arterial, } \\
\text { Hidronefrosis }\end{array}$ & $\begin{array}{l}\text { Casado } \\
2 \\
-\end{array}$ & $\begin{array}{l}\text { Casada } \\
\text { Hipertensión } \\
\text { arterial, Fibrilación } \\
\text { auricular, Accidente } \\
\text { vascular encefálico } \\
\text { hemorrágico e } \\
\text { isquémico }\end{array}$ \\
\hline Peso (kg) & 48.5 & 54 & 77 & 72.4 & 68 & 56 & 62.6 & 52 & 48 & 60 & 67.2 \\
\hline Talla (cm) & 152 & 165 & 175 & 168 & 170 & 160 & 165 & 165 & 168 & 172 & 165 \\
\hline Presión arterial sistólica & 99 & 100 & 111 & 152 & 95 & 126 & 149 & 126 & 100 & 150 & 126 \\
\hline Presión arterial diastólica & 72 & 60 & 74 & 100 & 50 & 72 & 89 & 71 & 65 & 100 & 75 \\
\hline Frecuencia cardíaca & 60 & 65 & 72 & 85 & 65 & 59 & 82 & 70 & 76 & 70 & 63 \\
\hline \multicolumn{12}{|l|}{ Síntomas } \\
\hline Disnea & & $\mathrm{X}$ & $x$ & & & $\mathrm{X}$ & & & $x$ & & $x$ \\
\hline Fatiga & $x$ & $x$ & & & & & & & & & \\
\hline Dolor precordial & & & & & & $\mathrm{x}$ & & & & & $\mathrm{x}$ \\
\hline Palpitaciones & & $\mathrm{X}$ & & $\mathrm{x}$ & & $\mathrm{X}$ & $\mathrm{X}$ & & & & \\
\hline Mareo & & & & & & $\mathrm{x}$ & & & $x$ & & $x$ \\
\hline \multicolumn{12}{|l|}{$\begin{array}{l}\text { Historia previa de paro cardíaco } \\
\text { Práctica de deporte competitivo }\end{array}$} \\
\hline \multicolumn{12}{|l|}{ Medicaciones } \\
\hline Betabloqueador & & $x$ & & $x$ & & $x$ & & & & & $x$ \\
\hline Antagonistas canales de calcio & & & & & & $x$ & & & & & $x$ \\
\hline Amiodarona & & & & & & & & & & & \\
\hline $\mathrm{IECA}^{\S}$ & & & & $\mathrm{x}$ & & $\mathrm{x}$ & & & $\mathrm{x}$ & & \\
\hline Hidroclorotiazida & & & & & & $x$ & & & & & \\
\hline Estatinas & & & & & & & & $\mathrm{x}$ & & & \\
\hline
\end{tabular}


Tabla 2 Parámetros ecocardiográficos de función diastólica

\begin{tabular}{|c|c|c|c|c|c|c|c|c|c|c|c|}
\hline \multirow{2}{*}{$\begin{array}{l}\text { Parámetros ecocardiográficos } \\
\text { de función diastólica }\end{array}$} & \multicolumn{11}{|c|}{ Identificación de los pacientes } \\
\hline & 2 & 3 & 4 & 5 & 6 & 7 & 8 & 10 & 11 & 12 & 13 \\
\hline$E$ & 0,9 & 0,47 & 0,98 & 0,7 & 0,88 & 0,69 & 0,51 & 0,61 & 0,69 & 0,6 & - \\
\hline$A$ & 0,48 & 0,2 & 0,51 & 0,5 & 0,51 & 0,66 & 0,69 & 0,44 & 0,57 & 0,44 & 0,21 \\
\hline$E / A$ & 1,9 & 2,4 & 1,9 & 1,4 & 1,7 & 1,0 & 0,7 & 1,4 & 1,2 & 1,4 & - \\
\hline$D T(m s)$ & 154 & 261 & 160 & 192 & 198 & 187 & 308 & 198 & 202 & 213 & - \\
\hline TRIV & 100 & 139 & 81 & 105 & 70 & 128 & - & 78 & 85 & 111 & 105 \\
\hline \multicolumn{12}{|l|}{ Doppler tisular } \\
\hline $\mathrm{S}(\mathrm{cm} / \mathrm{s})$ & 0,07 & 0,04 & 0,1 & 0,05 & 0,08 & 0,07 & 0,06 & 0,08 & 0,07 & 0,1 & 0,07 \\
\hline $\mathrm{e}^{\prime}(\mathrm{cm} / \mathrm{s})$ & 0,12 & 0,11 & 0,13 & 0,07 & 0,11 & 0,1 & 0,05 & 0,14 & 0,1 & 0,17 & 0,08 \\
\hline$a^{\prime}(\mathrm{cm} / \mathrm{s})$ & 0,05 & 0,04 & 0,07 & 0,06 & 0,08 & 0,07 & 0,11 & 0,09 & 0,07 & 0,09 & 0,02 \\
\hline$E^{\prime} / A^{\prime}(\gg 1)$ & 2,4 & 2,8 & 1,9 & 1,2 & 1,4 & 1,4 & 0,5 & 1,6 & 1,4 & 1,9 & 4,0 \\
\hline$E / e^{\prime}(>15)$ & 7,5 & 4,3 & 7,5 & 10,0 & 8,0 & 6,9 & 10,2 & 4,4 & 6,9 & 3,5 & - \\
\hline $\begin{array}{l}\text { Presión sistólica arterial } \\
\text { pulmonar }(\mathrm{mm} \mathrm{Hg})\end{array}$ & - & 29 & - & - & - & 21 & - & - & 23 & 29 & 38 \\
\hline
\end{tabular}

fibrilación auricular permanente con dilatación severa de la aurícula izquierda.

En cuanto a las evaluaciones electrocardiográficas, ninguno tuvo alteración del ritmo cardiaco. En los casos nuevos diagnosticados, 2 familiares cumplieron los criterios para el diagnóstico de miocardiopatía hipertrófica y 2 tenían ritmo de marcapaso.

En los parámetros ecocardiográficos evaluados, todos presentaron función ventricular izquierda y derecha normal. La medida de la aurícula izquierda se evaluó mediante dos métodos; al medir en eje paraesternal longitudinal, 2 casos tenían dilatación y diagnóstico de miocardiopatía hipertrófica. Cuando se aplicó la medida a través del volumen indexado con la superficie corpórea, se encontraron 3 casos, 2 de los cuales tenían aumento en eje paraesternal y uno (N. 12) aumento en el volumen indexado de la aurícula izquierda, con diagnóstico de hipertensión arterial de novo e hipertrofia ventricular excéntrica severa, sin criterios de miocardiopatía hipertrófica (tabla 2).

Los 4 miembros de la familia con criterios diagnósticos de miocardiopatía hipertrófica tuvieron presentación anatómica septal; la media del grosor septal fue de $24 \mathrm{~mm}$ (varió de 20 a 30 mm en la parte más gruesa). En ninguno se reportó obstrucción en el tracto de salida del ventrículo izquierdo en reposo o con la maniobra de Valsalva. La relación del grosor del segmento basal del septo y de la pared posterior fue mayor a 2 en todos los pacientes. Los parámetros de la función diastólica se relacionan en la tabla 3. Solo el paciente número 3 portador de miocardiopatía hipertrófica con cardiodesfibrilador, presentó patrón de llenado de tipo restrictivo.

La prueba de esfuerzo se llevó a cabo a 9 individuos; ninguno presentó arritmias supraventriculares o ventriculares durante el esfuerzo físico, hipotensión arterial o síntomas cardiovasculares durante el estudio (tabla 4). se interrumpió una prueba a causa de fatiga muscular y porque la paciente no alcanzó la frecuencia cardíaca máxima esperada. Dos pacientes no aceptaron la realización del examen, una por limitación funcional para la marcha (secuela de accidente cerebrovascular) y el otro por miedo de morir.
Se encontraron 3 casos sugestivos de isquemia miocárdica, uno de ellos con criterios diagnósticos de miocardiopatía hipertrófica, sin enfermedad coronaria significativa, ni gradiente en el tracto de salida del ventrículo izquierdo por el cateterismo cardíaco. Un paciente es hipertenso y no tiene hipertrofia ventricular izquierda. El otro individuo, tiene hipertrofía concéntrica del ventrículo izquierdo sin ser hipertenso (tablas 1 y 3 ).

Durante el estudio Holter de 24 horas ninguno desarrolló arritmias malignas, solo algunas extrasístoles ventriculares o supraventriculares aisladas. El paciente número 3 presentó trastorno en el segmento ST y en la onda T durante todo el examen y en el número 4 se observó trastorno difuso de la repolarización. La número 6 rechazó el examen por falta de disponibilidad de tiempo.

En el análisis, teniendo en cuenta el riesgo elevado de muerte súbita asociado a pacientes jóvenes y la posibilidad de afectar a varios miembros de una familia, se calculó el score-HCM Risk-SCD ${ }^{9}$ a todos los pacientes del estudio con criterios diagnósticos de miocardiopatía hipertrófica de acuerdo con el modelo de predicción de riesgo. Así, se obtuvo un riesgo calculado en el paciente número 3 de $4,9 \%$, en el 4 de $4,09 \%$, para el 8 de $2,18 \%$ y en el 13 de $5,09 \%$. Estos pacientes fueron evaluados por un electrofisiólogo quien sugirió estudio del potencial arritmogénico en aquellos que cumplieron los criterios diagnósticos de miocardiopatía hipertrófica e historia familiar de muerte súbita. En la paciente número 13 portadora de marcapaso unicameral por fibrilación auricular señaló el cambio por un cardiodesfibrilador y el cierre de la auriculilla izquierda.

\section{Discusión}

Pese a que la miocardiopatía hipertrófica familiar tiene un rasgo autosómico dominante, no se puede detectar hasta la etapa de desarrollo en la adolescencia. La hipertrofia puede producirse sin que se evidencien síntomas, lo que dificulta aún más el diagnóstico. 
Tabla 3 Parámetros ecocardiográficos de los pacientes estudiados

\begin{tabular}{|c|c|c|c|c|c|c|c|c|c|c|c|}
\hline \multirow[t]{2}{*}{ Parámetros ecocardiográficos } & \multicolumn{11}{|c|}{ Identificación de los pacientes } \\
\hline & 2 & 3 & 4 & 5 & 6 & 7 & 8 & 10 & 11 & 12 & 13 \\
\hline Ventrículo derecho $(\mathrm{mm})$ & 23 & 31 & 34 & - & 28 & 37 & 27 & 41 & 34 & 33 & 31 \\
\hline $\begin{array}{l}\text { Diámetro diastólico del ventrículo } \\
\text { izquierdo }(\mathrm{mm})\end{array}$ & 36 & 37 & 40 & 50 & 43 & 47 & 40 & 47 & 45 & 53 & 48 \\
\hline $\begin{array}{l}\text { Diámetro sistólico del ventrículo izquierdo } \\
\qquad(\mathrm{mm})\end{array}$ & 24 & 21 & 19 & 26 & 27 & 25 & 20 & 29 & 25 & 26 & 33 \\
\hline $\begin{array}{l}\text { Fracción de eyección del ventrículo } \\
\text { izquierdo (\%) }\end{array}$ & 62 & 65 & 83 & 80 & 69 & 78 & 80 & 69 & 76 & 82 & 67 \\
\hline Diámetro aurícula izquierda (mm) & 24 & 41 & 39 & 40 & 28 & 39 & 39 & 39 & 34 & 36 & 65 \\
\hline Volumen de aurícula izquierda & 15 & 45 & 28 & 13 & 21 & 25 & 24 & 26 & 18 & 43 & 97 \\
\hline \multicolumn{12}{|l|}{ Septo $(\mathrm{mm})$} \\
\hline Basal & 6 & 26 & 19 & 9 & 8 & 10 & 22 & 7 & 10 & 10 & 25 \\
\hline Medio & 8 & 30 & 20 & 8 & 7 & 7 & 19 & 7 & 8 & 11 & 18 \\
\hline Apical & 5 & 22 & 13 & 8 & 8 & 8 & 9 & 7 & 8 & 9 & 17 \\
\hline \multicolumn{12}{|l|}{ Lateral (mm) } \\
\hline Basal & 5 & 8 & 9 & 8 & 7 & 9 & 10 & 6 & 6 & 8 & 11 \\
\hline Medio & 7 & 11 & 7 & 8 & 8 & 7 & 9 & 9 & 6 & 10 & 11 \\
\hline Apical & 7 & 15 & 7 & 6 & 6 & 6 & 9 & 6 & 5 & 8 & 13 \\
\hline \multicolumn{12}{|l|}{ Inferior $(\mathrm{mm})$} \\
\hline Basal & 9 & 13 & 5 & 10 & 9 & 8 & 12 & 8 & 6 & 11 & 7 \\
\hline Medio & 9 & 19 & 9 & 6 & 9 & 7 & 11 & 6 & 6 & 11 & 7 \\
\hline Apical & 6 & 13 & 8 & 7 & 6 & 6 & - & 7 & 6 & 7 & 7 \\
\hline \multicolumn{12}{|l|}{ Anterior (mm) } \\
\hline Basal & 8 & 17 & 10 & 9 & 9 & 9 & 12 & 7 & 7 & 9 & 11 \\
\hline Medio & 8 & 15 & 8 & 8 & 8 & 9 & 11 & 7 & 6 & 10 & 11 \\
\hline Apical & 6 & 16 & 7 & 7 & 7 & 8 & 10 & 6 & 6 & 8 & 11 \\
\hline \multicolumn{12}{|l|}{ Posterior (mm) } \\
\hline Basal & 6 & 8 & 10 & 8 & 8 & 10 & 12 & 7 & 8 & 11 & 7 \\
\hline Medio & 8 & 9 & 9 & 7 & 9 & 7 & - & 7 & 7 & 10 & 7 \\
\hline Apical & 6 & 21 & 1 & 7 & 6 & 5 & - & 6 & 7 & 10 & 17 \\
\hline Índice de masa $\left(\mathrm{g} / \mathrm{m}^{2}\right)$ & 51 & 164 & 109 & 94 & 74 & 119 & 169 & 98 & 67 & 137 & 190 \\
\hline Grosor relativo & 0,44 & 0,43 & 0,45 & 0,36 & 0,4 & 0,47 & 0,60 & 0,43 & 0,36 & 0,42 & 0,31 \\
\hline $\begin{array}{l}\text { Gradiente tracto de salida del ventrículo } \\
\text { izquierdo }(\mathrm{mm} \mathrm{Hg})\end{array}$ & 3 & 5 & 3 & 4 & 4 & 5 & 4 & 4 & 5 & 4 & 6 \\
\hline $\begin{array}{l}\text { Gradiente tracto de salida del ventrículo } \\
\text { izquierdo }(\mathrm{mm} \mathrm{Hg}) \text { con maniobra de } \\
\text { Valsalva }\end{array}$ & 3 & 7 & 3 & 4 & 4 & 4 & 4 & 6 & 4 & 4 & 5 \\
\hline $\begin{array}{l}\text { Movimiento sistólico anterior de la } \\
\text { válvula mitral }\end{array}$ & no & no & no & no & no & no & no & no & no & no & no \\
\hline Insuficiencia mitral & no & si & no & no & no & si & no & si & no & si & si \\
\hline Grado de insuficiencia mitral & - & leve & - & - & - & leve & - & leve & - & leve & leve \\
\hline
\end{tabular}

Varias publicaciones sugieren el estudio genético como una herramienta útil para confirmar la presencia del gen y aumentar la casuística de la miocardiopatía hipertrófica en un grupo de pacientes asintomáticos, con historia familiar de muerte súbita y miocardiopatía hipertrófica asociada a hipertensión arterial. La guía de la Sociedad Europea de Cardiología $^{3}$, recomienda el estudio genético en todo paciente con miocardiopatía hipertrófica siempre que permita un estudio en cascada de los familiares (recomendación clase IB).

En la práctica clínica puede ser difícil el diagnóstico diferencial entre enfermedad cardíaca hipertensiva y miocardiopatía hipertrófica asociada a hipertensión arterial sistémica. La hipertrofia ventricular izquierda secundaria a sobrecarga de presión como ocurre en la hipertensión arterial, por lo general es simétrica; pero una pequeña parte de los pacientes puede presentar hipertrofia septal asimétrica. Algunos trabajos con ecocardiografía, a través de la evaluación de la deformación miocárdica (strain sistólico), demuestran su utilidad en el diagnóstico diferencial entre miocardiopatía hipertrófica e hipertrofia hipertensiva ${ }^{10,11}$. Es así como en este estudio se hallaron pacientes con hipertrofia ventricular izquierda asociada a hipertensión arterial que no llenaron los criterios de miocardiopatía 
Tabla 4 Prueba de esfuerzo y Holter de los pacientes estudiados

\begin{tabular}{|c|c|c|}
\hline Identificación de pacientes & Prueba de esfuerzo & Holter 24 horas \\
\hline 2 & Negativa & $\begin{array}{l}\text { No se encontraron arritmias ventriculares o } \\
\text { supraventriculares }\end{array}$ \\
\hline 3 & No realizada & $\begin{array}{l}\text { Algunas extrasístoles ventriculares aisladas y } \\
\text { supraventriculares aisladas; se observa transtorno } \\
\text { de ST/T durante todo el estudio }\end{array}$ \\
\hline 4 & Negativa & $\begin{array}{l}\text { No se encontraron arritmias ventriculares o } \\
\text { supraventriculares; se observa transtorno difuso } \\
\text { de la repolarización en diferentes áreas }\end{array}$ \\
\hline 5 & Negativa & $\begin{array}{l}\text { Algunas extrasístoles ventriculares aisladas y en } \\
\text { algunas ocasiones aparenta R/T }\end{array}$ \\
\hline 6 & Negativa & No realizado \\
\hline 7 & Negativa & $\begin{array}{l}\text { Se observan episodios de extrasístoles } \\
\text { supraventriculares aisladas y en cuatro ocasiones } \\
\text { taquicardia supraventricular autolimitada }\end{array}$ \\
\hline 8 & $\begin{array}{l}\text { Positiva para isquemia miocárdica y } \\
\text { negativa para arritmia cardíaca }\end{array}$ & $\begin{array}{l}\text { Algunas extrasístoles ventriculares aisladas y en } \\
\text { dos ocasiones pareadas; a veces presenta PR } \\
\text { corto, no hay arritmias supraventriculares }\end{array}$ \\
\hline 10 & Inconclusa & $\begin{array}{l}\text { No se encontraron arritmias ventriculares o } \\
\text { supraventriculares }\end{array}$ \\
\hline 11 & $\begin{array}{l}\text { Positiva para isquemia miocárdica y } \\
\text { negativa para arritmia cardíaca }\end{array}$ & $\begin{array}{l}\text { Algunas extrasístoles ventriculares aisladas y } \\
\text { supraventriculares aisladas y en tres ocasiones } \\
\text { pareadas y un evento de taquicardia } \\
\text { supraventricular }\end{array}$ \\
\hline 12 & $\begin{array}{l}\text { Positiva para isquemia miocárdica y } \\
\text { negativa para arritmia cardíaca }\end{array}$ & $\begin{array}{l}\text { Algunas extrasístoles ventriculares aisladas y } \\
\text { supraventriculares aisladas }\end{array}$ \\
\hline 13 & No realizada & $\begin{array}{l}\text { Ritmo de fibrilación auricular permanente de base } \\
\text { con episodios de respuesta ventricular rápida. }\end{array}$ \\
\hline
\end{tabular}

hipertrófica (pacientes N. 5, 7 y 12), dejando incertidumbre en el diagnóstico final. De esta forma, una gran limitación de la investigación es la no realización del estudio genético.

Otro factor fundamental como limitante de este estudio fue la no evaluación de la deformación miocárdica (strain sistólico) o el ecocardiograma de estrés con ejercicio. Un último inconveniente fue que no se pudieron efectuar todos los estudios diagnósticos en los pacientes involucrados ni pudieron evaluarse a todos los familiares de segundo grado por las razones citadas anteriormente, permitiendo aumentar la casuística del estudio.

Al finalizar los estudios de los familiares de la paciente índice, se descubrieron 4 miembros afectados con esta enfermedad, 2 de 3 en familiares de primer grado y solo en 2 de los 8 familiares de segundo grado estudiados. Los casos nuevos de miocardiopatía hipertrófica recibieron orientación acerca de la enfermedad. Estos pacientes requieren medidas preventivas con cambios en el estilo de vida, recomendaciones terapéuticas y seguimiento clínico para la prevención de eventos adversos.

\section{Responsabilidades éticas}

Protección de personas y animales. Los autores declaran que para esta investigación no se han realizado experimentos en seres humanos ni en animales.
Confidencialidad de los datos. Los autores declaran que han seguido los protocolos de su centro de trabajo sobre la publicación de datos de pacientes.

Derecho a la privacidad y consentimiento informado. Los autores declaran que en este artículo no aparecen datos de pacientes.

\section{Financiación}

Ninguna.

\section{Conflicto de intereses}

Ninguno.

\section{Agradecimientos}

A los pacientes por dedicar su tiempo disponible para que ese manuscrito fuera una realidad.

Al Dr. Ignacio Malabet, electrofisiólogo, por colaborar en el análisis de los casos.

A las auxiliares de enfermería Gelka Martínez y Yulimar Pertuz, por la colaboración absoluta.

A la enfermera Martha Cotes por facilitar la realización de los exámenes. 
A los funcionarios de Cardiostress Ltda. por la colaboración incondicional en la práctica de exámenes.

Al Dr. Oscar Giovanny Iglesias por su ayuda en la traducción al inglés.

\section{Anexo. Encuesta semiestructurada}

\section{RASTREO DE FAMILIARES DE UNA PACIENTE CON CARDIOMIOPATÍA HIPERTRÓFICA OBSTRUCTIVA (encuesta $n^{\circ}$ :}

NOMBRE:

FECHA I I I

EDAD: FECHA DE NACIMIENTO:

GENERO: ( ) MASCULINO

( ) FEMENINO

ESTADO CIVIL: NÚMERO DE HIJOS (y edad):

LUGAR Y DIRECCIÓN DE RESIDENCIA:

TELÉFONOS DE CONTACTO:

OCUPACIÓN:

RAZA (1).MESTIZA, (2).NEGRA, (3).CAUCÁSICO, (4).OTROS

1. Cuál es el grado de parentesco con la paciente índice?

2. Antecedentes familiares de enfermedad cardiaca. Cuáles?

enfermedades asociadas:

3. Practica algún deporte? Cuál? Cuántas veces a la semana?

4. Presenta síntomas? Cuáles?
( ) falta de aire
( ) fatiga
( ) angina
( ) dolor en el pecho

( ) palpitaciones

( ) mareo

( ) otros:

5. Ha tenido historia previa de paro cardíaco o síncope. (Si es así, cuándo y cuántas veces?) 
6. Presentó o presenta algún tipo de arritmia cardiaca? Cuál?

( ) taquicardia ( ) taquicardia auricular ( ) taquicardia supraventricular ( ) fibrilación auricular ( ) taquicardias ventriculares no sostenidas ( ) extrasístoles ventriculares

\section{Qué tratamiento ha recibido o le han realizado?}

( ) betabloqueadores ( ) verapamilo ( ) amiodarona ( ) técnicas quirúrgicas de miectomía o de alcoholización de la arteria coronaria septal ( ) marcapaso de doble cámara: marca

), ( )Otros, cuáles:

\section{Exámen físico:}

TA: $\mathrm{X} \_\mathrm{mmHg}$;

FC latidos por minuto;

Peso: $\mathrm{Kg}$;

Talla: $\mathrm{cm}$

\section{Bibliografía}

1. Elliott PM, Anastasakis A, Borger MA, Borggrefe M, Cecchi F, Charron P, et al. 2014 ESC Guidelines on diagnosis and management of hypertrophic cardiomyopathy: the Task Force for the Diagnosis and Management of Hypertrophic Cardiomyopathy of the European Society of Cardiology (ESC). Eur Heart J. 2014;35:2733-79.

2. Romero E, Marín B, Simarro E. Muerte súbita y deporte. Med Clin North Am. 1992;98:149-50.

3. Brugada J. Muerte súbita en la miocardiopatía hipertrófica. Rev Esp Cardiol. 1998;51:991-6.

4. Arteaga E, Ianni BM, Fernandes F, Mady C. Benign outcome in a long-term follow-up of patients with hypertrophic cardiomyopathy in Brazil. Am Heart J. 2005;149:1099-105.

5. Fernández A, Quiroga A, Ochoa JP, Mysuta M, Casabé $\mathrm{JH}$, Biagetti M, et al. Validation of the 2014 European Society of Cardiology Sudden Cardiac Death Risk Prediction Model in Hypertrophic Cardiomyopathy in a Reference Center in South America. Am J Cardiol. 2016;118: $121-6$.

6. Vriesendorp PA, Schinkel AF, Liebregts M, Theuns DA, Van Cleemput J, Ten Cate FJ, et al. Validation of the 2014 European Society of Cardiology guidelines risk prediction model for the primary prevention of sudden cardiac death in hypertrophic cardiomyopathy. Circ Arrhythm Electrophysiol. 2015;8:829-35.

7. Maron BJ, Casey SA, Chan RH, Garberich RF, Rowin EJ, Maron MS. Independent Assessment of the European Society of Cardiology Sudden Death Risk Model for Hypertrophic Cardiomyopathy. Am J Cardiol. 2015;116:757-64.

8. Wahr DW, Wang YS, Schiller NB. Left ventricular volumes determined by two- dimensional echocardiography in a normal adult population. J Am Coll Cardiol. 1983;1:863-8.

9. O'Mahony C, Jichi F, Pavlou M, Monserrat L, Anastasakis A, Rapezzi C, et al. A novel clinical risk prediction model for sudden cardiac death in hypertrophic cardiomyopathy (HCM Risk-SCD). Eur Heart J. 2014;35:2010-20.

10. Kato TS, Noda A, Izawa H, Yamada A, Obata K, Nagata K, et al. Discrimination of nonobstructive hypertrophic cardiomyopathy from hypertensive left ventricular hypertrophy on the basis of strain rate imaging by tissue Doppler ultrasonography. Circulation. 2004;110:3808-14.

11. Baratta S, Chejtman D, Fernández H, Ferroni FE, Bilbao J, Kotliar C, et al. Valor clínico de la utilización del strain rate sistólico en el estudio de distintas formas de hipertrofia ventricular izquierda. Rev Argent Cardiol. 2007;75:367-73. 